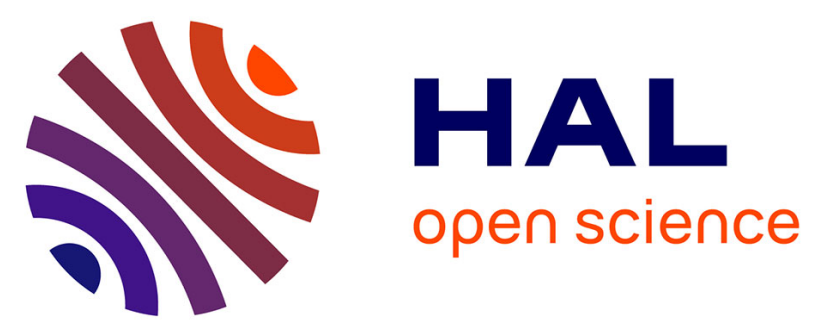

\title{
The nature of trapping sites and recombination centres in PVK and PVKPBD electroluminescent matrices seen by spectrally resolved thermoluminescence
}

Ireneusz Glowacki, Zbigniew Szamel

\section{- To cite this version: \\ Ireneusz Glowacki, Zbigniew Szamel. The nature of trapping sites and recombination centres in PVK and PVKPBD electroluminescent matrices seen by spectrally resolved thermoluminescence. Journal of Physics D: Applied Physics, 2010, 43 (29), pp.295101. 10.1088/0022-3727/43/29/295101 . hal- 00569655}

\section{HAL Id: hal-00569655 \\ https://hal.science/hal-00569655}

Submitted on 25 Feb 2011

HAL is a multi-disciplinary open access archive for the deposit and dissemination of scientific research documents, whether they are published or not. The documents may come from teaching and research institutions in France or abroad, or from public or private research centers.
L'archive ouverte pluridisciplinaire HAL, est destinée au dépôt et à la diffusion de documents scientifiques de niveau recherche, publiés ou non, émanant des établissements d'enseignement et de recherche français ou étrangers, des laboratoires publics ou privés. 


\title{
The nature of trapping sites and recombination centres in PVK and PVK-PBD electroluminescent matrices seen by spectrally resolved thermoluminescence
}

\author{
Ireneusz Glowacki $^{\text {a) }}$, Zbigniew Szamel ${ }^{\text {b) }}$ \\ Department of Molecular Physics, Technical University of Lodz, \\ 90-924 Lodz, Zeromskiego 116, Poland
}

a) Author to whom correspondence should be addressed, electronic mail: Ireneusz.Glowacki@p.lodz.pl

b) Present address: CSEM Centre Suisse d'Electronique et de Microtechnique SA Areal Rosental, R-1047.2, Mattenstr. 22, CH-4016 Basel

\begin{abstract}
Two electroluminescent polymer matrices: poly( $N$-vinylcarbazole) $(\mathrm{PVK})$ and PVK with $40 \mathrm{wt} \%$ of 2-tert-butylphenyl-5-biphenyl-1,3,4-oxadiazole (PBD) were studied using spectrally resolved thermoluminescence (SRTL) in temperature range 15-325 K. The comparison of the SRTL results with the electroluminescence (EL) spectra has allowed identifying the localized (trapping) sites and the radiative recombination centres present in the investigated matrices. In the neat PVK films deep traps with a depth about $200 \mathrm{meV}$, related to triplet excimers dominate, while in the PVK-PBD (40 wt $\%)$ blend films the traps that are related to triplet exciplexes formed by the carbazole groups and the PBD molecules dominate. Depth of the traps in the PVK-PBD blend is somewhat lower than that in the neat PVK. An analysis of the EL spectra shows that in the PVK and in the PVK-PBD blend the dominant radiative centres are singlet excimers and singlet exciplexes, respectively. However in the neat PVK some contributions of the triplet monomer and the triplet excimer states in the electroluminescence were also detected.
\end{abstract}

(Some figures in this article are in colour only in the electronic version) 


\section{Introduction}

Polymer light-emitting diodes (PLEDs) are of great current interest due to their potential applications in large-area and flexible displays, which can be fabricated at room temperature, by inexpensive solution-processing methods like spin-casting, screen-printing or inkjet-printing $[1,2]$. To get optimal efficiency and device lifetime, the injection and transport of holes and electrons must be balanced, so that similar concentrations of these two kinds of charge carriers are achieved. In case of polymers which contain the radiative recombination centres, the single-layer devices can be prepared in simple way but the active layer should assure both hole and electron transport besides light emission [3].

Poly(N-vinylcarbazole) (PVK), a non-conjugated polymer with a luminescent carbazole side groups, is a well-known and is often used as the optically active material in different optoelectronic devices, due to its excellent film-forming, relatively good hole-transporting properties, and large separation between highest occupied molecular orbital-lowest unoccupied molecular orbital (HOMOLUMO) [4]. PVK is widely utilized especially in the fabrication of blue light emitting diodes in place of conjugated polymers, which have weak emission in blue region due to their long $\pi$-conjugation and relatively low fluorescence quantum yields $[3,4]$. Since the pure PVK has poor electron-transporting properties, oxadiazole derivatives such as 2-tert-butylphenyl-5-biphenyl-1,3,4-oxadiazole (PBD) (figure 1) is often added to PVK [4,5]. The PVK-PBD blend is particularly used as host for the phosphorescence guest materials at low concentration because PVK has high triplet exciton energy that prevents crossing of the triplet exciton back from the phosphor to the host triplet state [4].

Several groups have reported electroluminescence spectra of pure PVK [6-10]. In all the cases, a broad and unstructured emission band extending from $350 \mathrm{~nm}$ to over $650 \mathrm{~nm}$, with a dominant broad maximum at 420-445 nm, was observed [6-9]. The emission at $c a$ 420-430 nm comes from the low energy singlet excimer (full overlap carbazole units). The shorter wavelength shoulder at 370-380 nm and some contribution of the band at $350-360 \mathrm{~nm}$ can be related to the higher energy singlet excimer and to isolated carbazole groups, respectively [7-9]. All the EL maxima detected for PVK are redshifted by ca $10 \mathrm{~nm}$ in comparison to the photoluminescence (PL) spectra. Recently Angelo et al. [8] published broad EL spectra of the neat PVK, where apart from the dominant band at $c a 410 \mathrm{~nm}$ also the bands at $450-460 \mathrm{~nm}, 480 \mathrm{~nm}, 500-550$ and over $600 \mathrm{~nm}$ can be distinguished. However the 
authors have not discussed the origin of these additional bands in EL spectra. Also Qian et al. [9] reported a broad EL spectrum for single layer PVK devices in the range 380-650 nm with a dominant peak at $420-430 \mathrm{~nm}$ and a minor one at $590 \mathrm{~nm}$ with additional very weak shoulder at $\sim 630 \mathrm{~nm}$. The long-wavelength emission with weak intensity was also observed in the PL measurements either at 80 $\mathrm{K}$ or at room temperature and the authors related these bands to the triplet excimers in the PVK films [9]. They found that probability of the singlet and triplet exciton state forming can be changed by using of different solvents for PVK solution preparation or PVK with different molecular weight. Although PVK has non-conjugated backbone but stacking of side carbazole groups can create conjugated system with the two types of excimers as it is discussed above. The authors argued that the probability of triplet formation in conjugated polymers increases with decreasing effective conjugation length. When the effective conjugation length decreases the intersystem crossing rate increases and the population of triplet excitons those come from singlet excitons increases due to the lower exciton dissociation probability. This conclusion was confirmed by a quantum chemical calculation made by Belionne et al. [11]. It is known that position of the HOMO/LUMO levels is not only determined by the chemical structure of the molecule itself but also the electronic polarisation of its surrounding. For polymers, the effective conjugation length also affects the position of the HOMO/LUMO levels. Change of the molecular conformation will lead to a fluctuating surrounding and to fluctuating conjugation length. Qian et al. [9] found that the partial overlap conformation has lower triplet/singlet ratio as compared to the full overlap conformation. The influence of molecular conformation of polymer chains on its triplet formation probability has attracted attention of several researcher groups.

Interesting conclusion can be drawn from the recently published EL spectra for PVK spin cast layer obtained after previous irradiation of the solution with UV light [12]. With increasing UV light irradiation time, the intensity of the emission about $600 \mathrm{~nm}$ increased, while the emission band at 420 $\mathrm{nm}$ decreased and red-shifted. The authors explained these effects by increasing population of the full overlap carbazole excimers. However they also indicated that several different photophysical and photochemical processes can occur in PVK solution as a result of UV light irradiation, like conversion from partially to full overlap carbazole units, cross-linking of PVK through the carbazole groups with 
contribution of oxygen, photoscission of PVK, or debonding of carbazole group from a PVK chain [12].

It is worth to note that up to now the excimeric phosphorescence in the PVK films was related to the emission in the range $480-530 \mathrm{~nm}$; however lower energetic tail down above $650 \mathrm{~nm}$ was observed in both phosphorescence and EL spectra [6,8]. This emission bands about $590 \mathrm{~nm}$ and above $600 \mathrm{~nm}$ were attributed to several different mechanisms, such as aggregation, [7,9] electroplex [3,9] or phosphorescence $[13,14]$. In contrast to PVK, for which photophysical properties were extensively studied since many years, the PVK-PBD system was investigated much less intensively. The EL spectra for the PVK-PBD blends have dominant peak at $430 \mathrm{~nm}$, which is red-shifted by $c a 5 \mathrm{~nm}$ in comparison to the PL spectrum; this effect is explained by formation of the exciplex states. It was also shown that the EL spectra have some long-wavelength contribution dependent on composition of the blends and on applied electric field [4,10].

The energy transfer and the charge carrier trapping play an important role in the optoelectronic materials taking into account their further application. Traps in disordered media are commonly considered as localized states. Trapped charge carriers may be released after a retention time and will become free or may recombine with carriers of the opposite sign. When the release rate is higher than the recombination rate, the localized state is a trap, while for dominant recombination rate the localized state forms a recombination centre. It means that the same state may act as the recombination centre or as the trap, depending on certain parameters, like temperature or a ratio of minority to majority carrier concentrations. It is well known that the traps can reduce the carrier drift mobility and disturb the internal field distribution. Trapped charge carriers can quench emissive optical excitations and thus restrict the EL efficiency of organic materials.

One of the most direct methods of investigation of the trapping phenomena is thermoluminescence (TL). Thermoluminescence is observed when a sample of i.e. a polymeric material is heated after excitation at low temperature. The excitation generates charge carries, most of them recombine in very short time, but some of them gain enough energy to escape the geminate recombination. These charge carriers, after thermalization, can stay in the localized sites-traps. The subsequent heating causes their detrapping, and if they recombine radiatively, a thermoluminescence 
is detected [14-16]. The TL investigations for PVK [14] and for PVK with $30 \mathrm{wt} \%$ PBD blend [17] were reported previously but the spectra were obtained in the narrower spectral range $(350-580 \mathrm{~nm})$ because the used photomultiplier had the sensitivity maximum in blue range. The indicated above literature review showed that many reports devoted the EL and PL investigation for PVK in the spectral range above $500 \mathrm{~nm}$ are not able to provide clear explanation of this emission origin. This reason motivated us to taking trial of the thermoluminescence technique use in extended spectral range for obtaining more information about the emission mechanism in the polymer system.

In the present study, we report investigations of spectrally resolved thermoluminescence (SRTL) in a broad spectral range $(350-950 \mathrm{~nm})$ for the neat PVK and for the PVK with 40 wt \% PBD blend films. An analysis of the TL, PL and EL spectra has allowed to characterize the excited states, the trapping/transport sites and the recombination centres.

\section{Experimental}

The PVK and PBD were obtained from Sigma-Aldrich and were used as received. Chemical structure of the materials are shown in figure 1. For the TL measurements films of PVK and PVK-PBD (40 wt $\%)$ were cast from chlorobenzene solutions onto stainless steel substrates at room temperature.

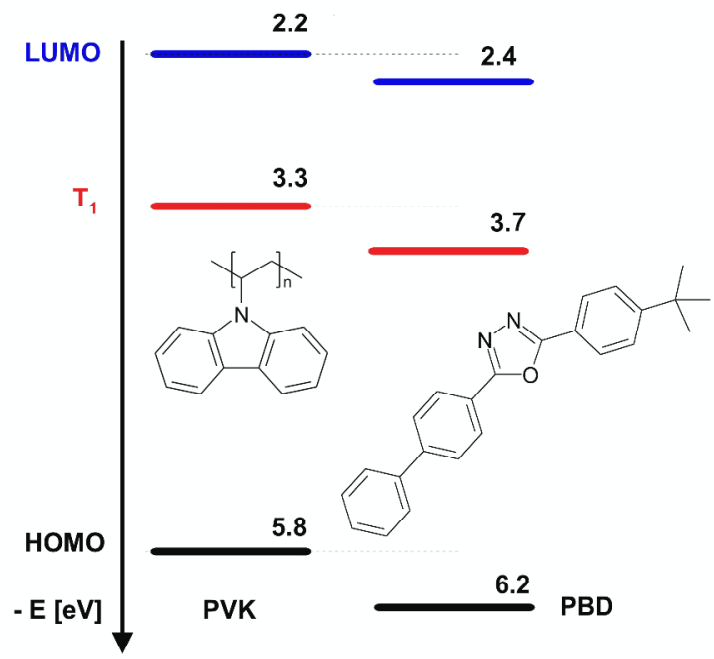

Figure 1. Chemical structures of investigated materials and diagram of energetic levels. The values of energetic levels according to ref. 4 . 
Thicknesses of the films were between 5-8 $\mu \mathrm{m}$ range. To remove the residual solvent, the films were dried at $423 \mathrm{~K}$ for several hours in the nitrogen atmosphere. For TL experiment the sample was placed in a vacuum chamber (closed-cycle cryogenic system APD Cryogenics, type Displex) and squeezed between a thermostated stage and a sapphire plate, with the aid of a brass frame anchored thermally to the stage. The TL measurements were performed in the temperature range from 15-325 K. The sample was photo excited for 10 minutes using a mercury lamp HBO-200 at $15 \mathrm{~K}$. We used band pass filter in front of the mercury lamp with $\lambda_{\max .}=365 \mathrm{~nm}$ yielding light output power of about $2.5 \mathrm{~mW} / \mathrm{cm}^{2}$. Then the sample was heated gradually with the rate of $7 \mathrm{~K} / \mathrm{min}$. Light emitted during the SRTL experiment was detected using specially designed optical system consisting of custom made optical collector, optical fiber, Micro HR Imaging Spectrograph and a CCD camera (Horriba Jobin-Yvon). The system has flat sensitivity response in the whole wavelength range $350-950 \mathrm{~nm}$. The analysis program of the TL measurement system creates TL spectra in the form of 3-D plots (luminescence intensity versus temperature and wavelength) or in a form of TL map. From such map one can extract the monochromatic TL curve for any chosen wavelength or the luminescence spectrum at any chosen temperature.

The activation energies $\left(E_{a}\right)$ of the detrapping processes were determined from the so-called "partial heating" experiments. It consisted of recording the emitted light during subsequent heating/cooling cycles with progressively increasing maximal temperature in each cycle. To monitor the luminescence intensity during the partial heating experiment, we used the photomultiplier tube (Thorn EMI type 9789 QB) in the optical system.

The same detection system as for the SRTL experiment was also used for spectral analysis of the electroluminescence of the single-layer PLEDs. The PLED devices were produced in the following way. We spin coated $30 \mathrm{~nm}$ thick layer of poly(3,4-ethylenedioxythiophene) poly(styrenesulfonate) (PEDOT-PSS) onto a clean indium tin oxide (ITO) coated glass substrate (ITO resistivity $15 \Omega /$ sq.), purchased from Präzisions Glass and Optik $\mathrm{GmbH}$ and then annealed at $423 \mathrm{~K}$ for $1 \mathrm{~h}$ in nitrogen atmosphere. Then the emitting layers, either PVK or PVK-PBD (40 wt \%) layer of thickness about $120 \mathrm{~nm}$ were spin coated and the samples were again annealed at $443 \mathrm{~K}$ for $2 \mathrm{~h}$. Finally we evaporated 
$5 \mathrm{~nm}$ thick $\mathrm{LiF}$ and $100 \mathrm{~nm}$ thick $\mathrm{Al}$ as contacts on top of polymeric layer using Edwards system (model Auto 306).

Steady-state photoluminescence (PL) experiments were performed using Jobin-Yvon Spectrofluorimeter (model Fluorolog FL3-11). For the PL measurements, we spin coated PVK or PVK-PBD thin films of $c a .150 \mathrm{~nm}$ onto quartz substrates. Thickness and roughness of the sample surface were determined by AFM (NT-MDT AFM model SOLVER PRO).

\section{Results and discussion}

\subsection{Photoluminescence and electroluminescence}

The PL spectra of the films of PVK and PVK-PBD (40 wt \%) obtained after excitations at $\lambda=337 \mathrm{~nm}$ are shown in figure 2. It should be noted that absorption bands of PVK and PVK-PBD blend in the wavelength range 300-360 nm are similar (see an inset in figure 2). The PL maximum for PVK at $c a$ 410-415 nm with short-wavelength shoulder, is due to the emission from two different types of the carbazole singlet excimers and also from isolated carbazole groups (at 350-360 nm). Similar spectra for PVK were reported by Klöpffer and Fischer [18] and by Bässler [19], who have argued that in PVK the traps are neutral and probably connected with carbazole dimers yielding excimer states. Johnson and Good [20], Itaya et al. [21] and quite recently Qian et al. [9] suggest an existence of two types of excimer species in PVK: the lower energy (corresponding to the emission peak at ca 410-420 nm due to so-called "sandwich"-like full-overlap excimers) characterized by a coplanar arrangement of two neighbouring carbazole units, and the higher energy (corresponding to the emission peak at $c a$ $380 \mathrm{~nm}$ ) "second excimers" (partially-overlap excimers) created by the overlapping of only one benzene ring from two contacting carbazole groups in the $t t$ conformation of the syndiotactic sequence in PVK.

The PL maximum for the PVK-PBD blend is red-shifted by about $15 \mathrm{~nm}$ in comparison to the PL maximum of neat PVK and also of pure PBD, for which the PL maximum appears at $390 \mathrm{~nm}$ [22]. It is well-known that the oxadiazole and the carbazole groups have a tendency to form the excited state complexes. Therefore the PL maximum at $c a 425-430 \mathrm{~nm}$ has been assigned to the exciplexes, which are created rapidly after the excitation of the PVK-PBD matrix [10,22]. Such interpretation was 


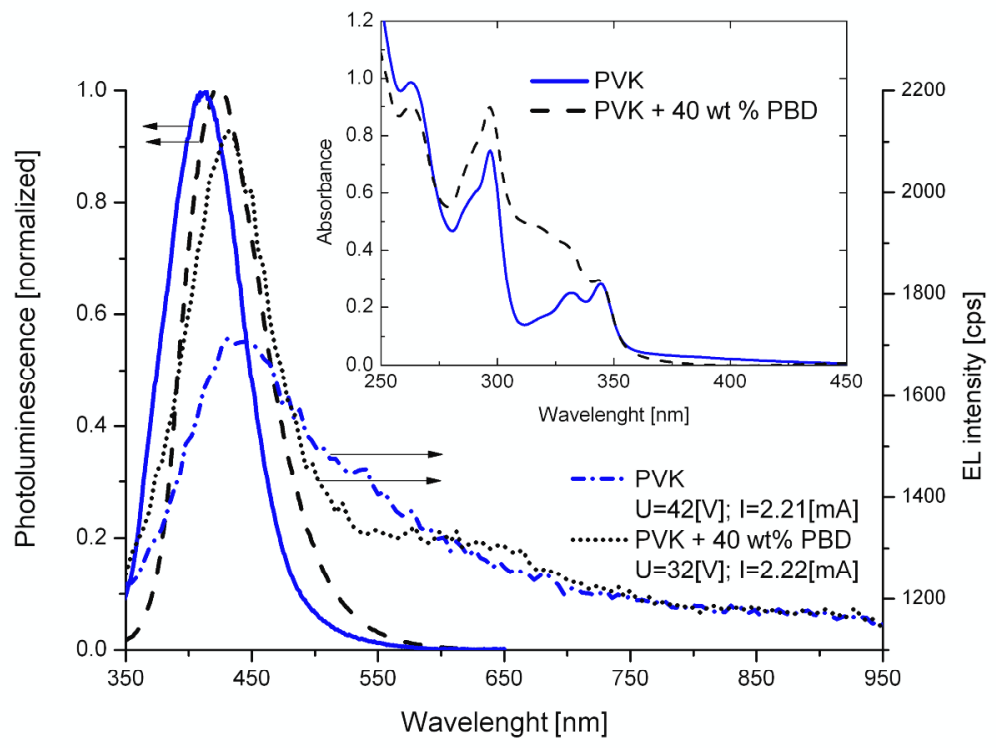

Figure 2. Normalized photoluminescence spectra of PVK and PVK-PBD (40 wt \%) thin films under excitation at $337 \mathrm{~nm}$ and electroluminescence spectra of single layer PLEDs prepared based on PVK and PVK-PBD (40 wt \%) blend, measured for the same current density, 22 $\mathrm{mA} / \mathrm{cm}^{2}$. The inset shows the corresponding absorption spectra.

supported by an observation that the PL intensities of the PVK-PBD blends increase with an increase in PBD content. This indicates that PBD is not an electron acceptor which would quench the PL emission of PVK. Formation of exciplexes in PVK-PBD (40 wt \%) films was observed by several groups using steady state and time-resolved photoluminescence spectroscopy $[10,22,23]$. Negres et al. [23] used time-resolved photoinduced absorption measurements at $77 \mathrm{~K}$ and room temperature to show that in PVK-PBD host blend, an additional excited-state (exciplex) is formed and charge trapping plays an important role in excited-state dynamics. It was observed by probing at longer wavelengths $(480,532$ and $580 \mathrm{~nm})$ that $35 \%$ of primary photoexcitation evolve into charge trapping states. The trapping states in a film of PVK-PBD blend have an emissive character at $480 \mathrm{~nm}$ but become absorptive at longer wavelengths [23].

The EL spectra of neat PVK and PVK-PBD (40 wt \%) devices are shown in figure 2. It should be noted that the EL spectra are obtained for the same current density $22 \mathrm{~mA} / \mathrm{cm}^{2}$ by adjusting the bias voltage. It is important to compare the contributions of different kinds of excited states in the EL spectra for the two matrices; especially when it is known that without dopants the EL intensity is low. 
For PVK, we observed a broad and unstructured emission from 350-750 $\mathrm{nm}$ along with a dominant broad maximum at $420-445 \mathrm{~nm}$. As mentioned in the introduction, the peak at $420-430 \mathrm{~nm}$ results from a low energy singlet excimer (full overlap carbazole units). The shorter wavelength shoulders at 370-380 $\mathrm{nm}$ and at 350-360 $\mathrm{nm}$ can be related with the higher energy singlet excimer and with isolated carbazole groups, respectively [7,9]. The EL maximum is red-shifted by $c a 10 \mathrm{~nm}$ in comparison with the PL spectra. However, apart from the dominant peak in the EL spectra one can observe a weak, longer wavelength bands with gradually decreasing intensity. These bands are strongly overlapping each other but they are located in the regions: $470-500 \mathrm{~nm}, 530-550 \mathrm{~nm}$ and over $600 \mathrm{~nm}$. Similar EL bands were observed in PVK by several research groups [6-9] but only Qian et al. [9] discussed these long-wavelength bands. It was suggested that these bands around $600 \mathrm{~nm}$ may arise from the excimer triplet states. It seems that these emissions can be related to different extended staking of carbazole groups (entanglements of chains and some aggregates of carbazole groups) or other unknown species present in the PVK films.

The EL spectrum of the device with PVK-PBD (40 wt \%) blend has dominant peak at $430 \mathrm{~nm}$, which is red-shifted by $c a 5 \mathrm{~nm}$ in comparison with the PL spectrum. It also shows some contributions of both short- and long-wavelength emissions. The rising edge of EL maximum is very similar both in PVK and PVK-PBD systems but considerable difference for longer wavelength can be observed. Addition of PBD to PVK causes a significant enhancement of the dominant EL band with maximum at $430 \mathrm{~nm}$. This effect is caused mainly because of improved balance of holes and electrons in the PVKPBD layer. The PBD molecules create transport channels for electrons. Moreover they can also form exciplexes with carbazole groups, which in turn can work as radiative centres rising the EL emission band at $430 \mathrm{~nm}$. One can also see that introduction of PBD to PVK causes some increase of EL emission in the range $600-750 \mathrm{~nm}$ and simultaneously a significant reduction of the EL intensity in the range 470-600 $\mathrm{nm}$. To explain the influence of PBD on emission spectra of PVK, extended thermoluminescence (TL) studies were performed.

\subsection{Thermoluminescence}


The 3D plot of the SRTL spectrum for neat PVK film photoexcited at $15 \mathrm{~K}$ is shown in figure 3, and the monochromatic TL curves and spectrally resolved spectra at temperatures corresponding to TL maxima are shown in figure 4. As one can see from the TL map, the chosen wavelengths $(500 \mathrm{~nm}$ and $530 \mathrm{~nm}$ ), at which the monochromatic TL curves were extracted, correspond to the wavelengths at

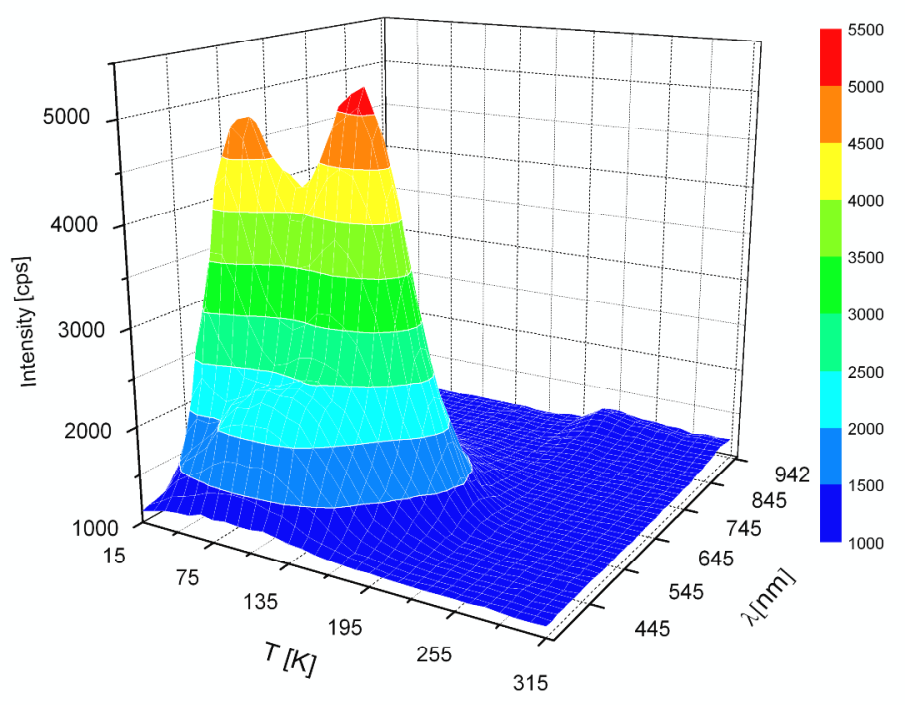

Figure 3. Three-dimensional thermoluminescence spectra for neat PVK film registered under the heating rate $7 \mathrm{~K} / \mathrm{min}$ after excitation by HBO-200 lamp with band filter at $15 \mathrm{~K}$.

which the two maxima on TL map appear. One can also see that both monochromatic TL curves have two broad maxima. One maximum is below $50 \mathrm{~K}$ and the second one is around $125-135 \mathrm{~K}$. Both maxima have similar intensities but their relative intensities are different. In the spectrum for $\lambda=530$ $\mathrm{nm}$, high-temperature maximum is slightly higher than the low temperature one. On the other hand, in the spectrum for $\lambda=500 \mathrm{~nm}$ this relationship is reversed. This indicates that upon heating, the trapped charge carriers are subsequently liberated from two different trapping sites of similar densities.

Simultaneous spectral analysis of the light emitted during the TL experiment allows us to identify the origin of radiative recombination processes giving rise to the TL signal. The spectrally resolved luminescence (SRL) recorded at different temperatures during the TL experiment (see the TL map in figure $4 \mathrm{a})$ reveals that the luminescence extends over entire visible range. The two SRL spectra detected close to the TL maxima, i.e. at $50 \mathrm{~K}$ and at $130 \mathrm{~K}$, are shown in figure 4c. In both cases one can distinguish two broad maxima: one weaker at the short wavelength range around $400 \mathrm{~nm}$ and the 

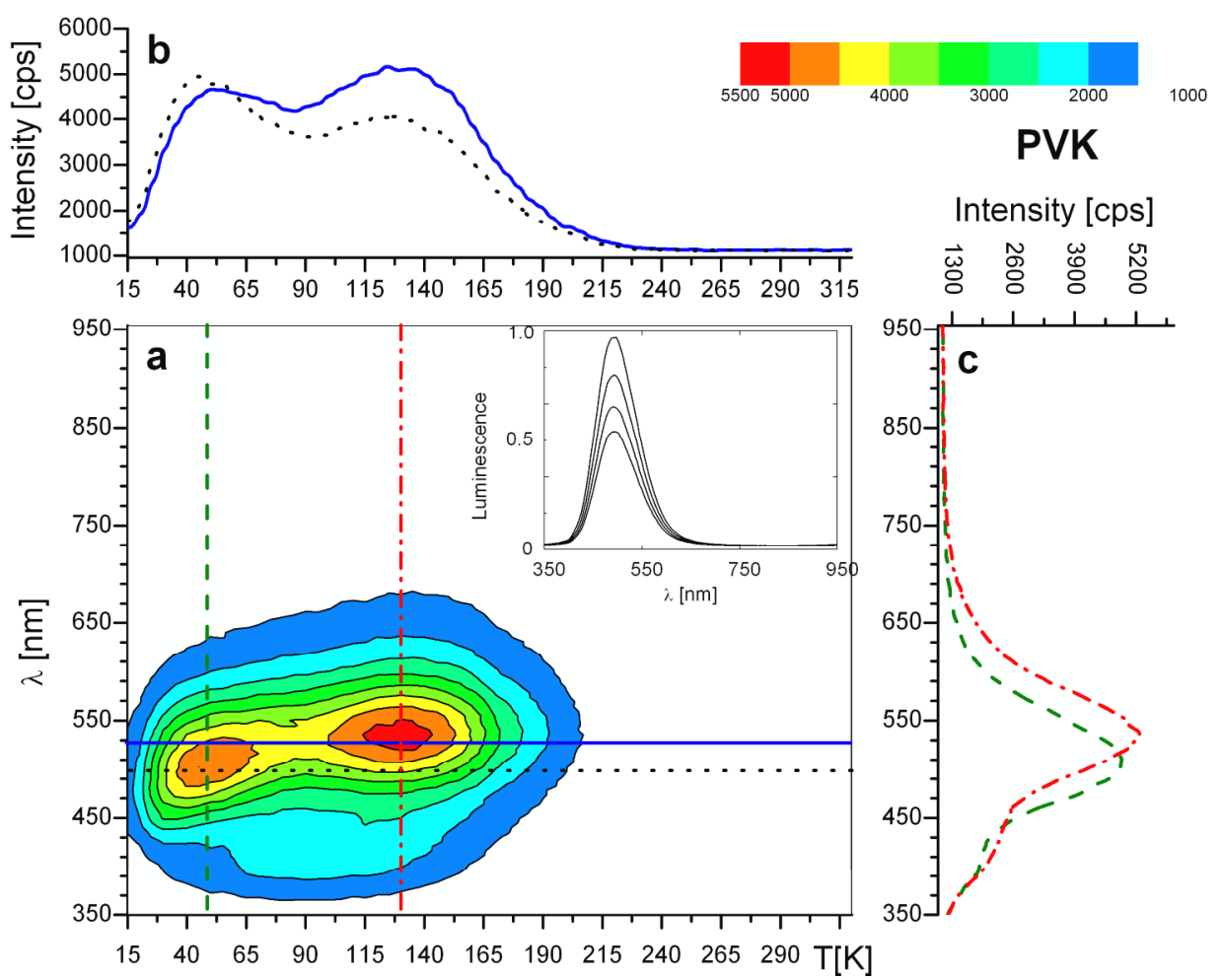

Intensity [cps]
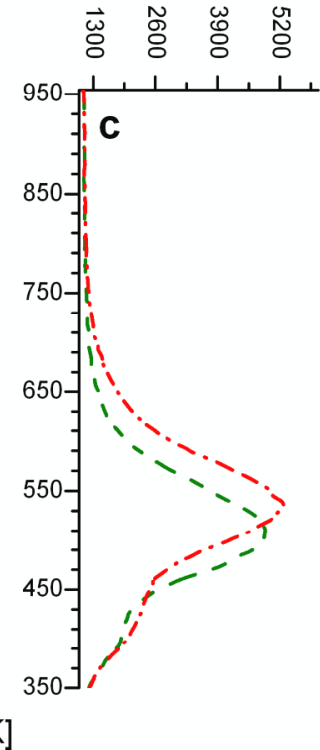

Figure 4. TL spectrum map for neat PVK film (a) with separated monochromatic TL curves (b) and spectral resolved curves (c). Marked lines on TL map indicate: chosen wavelengths (horizontal lines) and chosen temperatures (vertical lines). The inset presents spectra of isothermal luminescence decay at $15 \mathrm{~K}$ before TL run.

second dominating band at longer wavelength, which shifts its position from $c a 500 \mathrm{~nm}$ at low temperature to $c a 530 \mathrm{~nm}$ at high temperatures. Additionally, before the TL run, the isothermal luminescence spectra were recorded at $15 \mathrm{~K}$ in the time interval from 10 seconds to several minutes after turning off the excitation. The spectra for PVK shown in the inset in figure 4a have broad band in the range 400-600 nm with a maximum at $c a 490 \mathrm{~nm}$ and their shapes do not change in time. It was found [14] that the isothermal phosphorescence spectra for PVK films registered in range 5-140 K show bands at 425-430 $\mathrm{nm}$ and 450-460 nm, which are well observed only at temperatures $5 \mathrm{~K}$ and $20 \mathrm{~K}$ and they disappear at higher temperatures. At the same time the broad band at lower wavelengths shifts from $490-500 \mathrm{~nm}$ to $520-530 \mathrm{~nm}$ with increasing temperature. Similar evolution of the phosphorescence spectra for PVK upon the temperature increase was observed earlier by Burkhart and Chakraborty [13]. It has been explained as a change of photoexcited states in PVK from monomeric triplet states at low temperatures to excimeric ones at higher temperatures. They observed a temperature dependent discontinuity (the Arrhenius graph) of the rate constant for phosphorescence 
decay in the range $40-55 \mathrm{~K}$. The authors have concluded that the increased rate of decay of nonexcimeric triplets is due to the trapping at excimer-forming sites.

From above analysis it can be concluded that both the monomeric and the excimeric states of carbazole groups are involved in the radiative recombination during the TL processes. At temperatures below $50 \mathrm{~K}$ the monomeric triplet states dominate $(480-500 \mathrm{~nm})$ and at higher temperatures the excimeric triplet states $(520-530 \mathrm{~nm}$ ) prevail. The weak emission in the range $370-420 \mathrm{~nm}$ (see figure 4c) can be due to the contribution from both monomeric and excimeric singlet states of carbazole groups $[9,13,14]$. We observed a shift of the band maximum from $480-500 \mathrm{~nm}$ to $520-530 \mathrm{~nm}$ (see figure 4c). This evolution of the emission spectra with increasing temperature can be explained by the change of relative conformation of carbazole groups due to molecular relaxation activated at higher temperatures.

In order to determine activation energies of the detrapping processes, which is a measure of trap depth, the partial heating experiments were performed. The results obtained for PVK (not shown here) indicate the presence of two main trapping levels: around $50 \mathrm{meV}$ and $200 \mathrm{meV}$ corresponding to the low and high-temperature TL maxima, respectively. Similar value of $E_{a}$ in the high temperature range (200 meV) was obtained in a TL experiment performed on PVK film in the temperature range between the liquid nitrogen and room temperature $[14,24]$. The activation energy of phosphorescence obtained from temperature dependent isothermal measurements of lifetime of triplet states in the temperature range $10-40 \mathrm{~K}$ is $19 \mathrm{meV}$ [14]. This agrees well with a value of $21 \mathrm{meV}$ obtained by Burkhart and Chakraborty [13] from an analogous measurements. These values are at least twice lower than the value ca $50 \mathrm{meV}$ obtained from the TL partial heating experiments which correspond to the lowtemperature TL maximum and are related to shallow traps in PVK film. It should be noted that the shallow traps with the depth much lower than $50 \mathrm{meV}$ can not be detected in TL experiment, which starts at $15 \mathrm{~K}$. Because the charge carriers are already released from such shallow traps soon after the photoexcitation before the start of the heating run.

The TL maxima are directly related to the charge carrier detrapping; however it does not mean that the activation energy values estimated from the partial heating of $\mathrm{TL}$ always correspond to the depth of the traps. In polymers, some low polarized energy of the trapping sites can be formed by 
"physical defects" such as cavities resulting from the specific arrangement of polymer chains, socalled "conformational traps". Thus, the TL maxima often correspond well to the onset of molecular motions, what affords evidence for a significant contribution of the molecular motions to the charge carrier detrapping process. Such mechanism is called as the "wet dog" effect [25]. Akuetey and Hirsh [26] based on an analysis of dielectric measurements for PVK made by Pochan et al. [27], have found that the $\gamma$-relaxation in this polymer extrapolated to the $\mathrm{mHz}$ frequency range should appear at around $130 \mathrm{~K}$. This frequency range corresponds to an equivalent frequency of thermally stimulated processes, if the heating rate is a few $\mathrm{K} / \mathrm{min}$ [26]. It means that the detrapping which gives rise to the TL maximum at around $130 \mathrm{~K}$ could be induced by the $\gamma$-relaxation in PVK. Also the low-temperature TL maximum might be correlated with the $\delta$-relaxation in PVK, which is attributed to a wagging motion of the carbazole groups [27]. On the other hand, the activation energies of molecular relaxations determined from the dielectric measurements are higher in comparison to those estimated from the TL partial heating experiments, e.g. values of $390 \mathrm{meV}$ in the relation to $200 \mathrm{meV}$ and nearly $100 \mathrm{meV}$ to $50 \mathrm{meV}$ for $\gamma$-relaxation and $\delta$-relaxation respectively [27]. This is because only an onset of molecular motions may be sufficient to facilitate detrapping of the localized charge carriers $e . g$. by lowering the potential barriers of such trapping sites or by decreasing the distance between the neighbouring transport sites, and therefore entire molecular relaxation process does not need to be involved. Similar effect was reported also for other polymers [25].

Arkhipov et al. [28] and Fishchuk et. al. [29] proposed that TL in polymers is controlled by thermally assisted hopping relaxation of charge carriers. In this approach the TL spectrum provides information on charge carriers from tail states of intrinsic density-of-states (DOS) distribution and from deeper traps. In disordered media deep traps are extrinsic localized states that differ from the majority of hopping (transport) states where they require substantially higher energy input to release the charge carriers back to the intrinsic DOS. Therefore it is not clear, which sites play a role of traps and which are regular transport states. For instance, hole or electron transport proceeding via one kind of charge transporting material would be affected by trapping on a molecule of another kind, provided that the latter has a lower ionisation potential or a higher electron affinity, respectively. It has been mentioned that traps can be also of structural origin like dimers or aggregates, what can be observed in 
many polymer systems and also in case of PVK and PVK-PBD blend. Therefore the interpretation of TL phenomena in disordered organic materials with hopping charge transport is not as straightforward as in inorganic crystalline materials. According to Fishchuk et al. [29] the activation energy of the dominant TL maximum is proportional to the effective energetic disorder parameter $\sigma_{\text {eff. }}$ Two models, the effective medium approach proposed by Movaghar [30] and the variable range hoping described by Arkhipov et al. [31] for energetic relaxation of carriers, could be applied for description of the population of localized sites taking part in the process of low-temperature energetic relaxation of the photogenerated carriers. According to these theories, the energetic relaxation of generated particles in the zero-temperature limit occurs through a non-activated downward hopping with the decay of mean energy according to the expression [32]:

$$
E_{R}=\sigma\left[3 \ln \ln \left(\frac{t}{t_{0}}\right)\right]^{1 / 2}
$$

where: $E_{R}$ is the mean energy of the charge-carrier packet relative to the centre of the DOS distribution, $\sigma$ is the width of the DOS distribution, $t$ is the time, and $t_{0}$ is the dwell time of carrier at a lattice site without disorder. In this case (according to ref. 32) $t_{0}$ is accepted as $10^{-13} \mathrm{~s}$.

The low-temperature relaxation process described by the above expression practically saturates in experimental time scale of minutes, thus the parameter $\sigma$ can, at least roughly, be estimated from the activation energy at the TL peak maximum, assuming that the latter corresponds to the mean energy of the relaxed charge carrier packet, $E_{R}$. It should be noted that in such reasoning one should also take account the fact that the thermal release of carriers occurs to the effective transport energy level that normally is located below the centre of the DOS. Nevertheless, the parameter $\sigma$ is roughly proportional to the activation energy measured in the TL maximum and corresponding to the temperature of the TL maximum as the mean activation energy measured by partial heating TL experiment [29]. It means that from our partial heating TL results, in case of neat PVK film the parameter $\sigma$ reaches the value of $0.2 \mathrm{eV}$. Taking the value of $\sigma$ equal to $0.2 \mathrm{eV}$ and $t$ in the range from $100-1000 \mathrm{~s}$ after terminating the excitation inserting the data to the above expression we can obtain information that the charge carrier would be localized by approximately $3 \sigma=0.6 \mathrm{eV}$ below the DOS centre. It agrees well with a value of 
$0.6 \mathrm{eV}$ obtained by Bässler [33] and with $0.5 \mathrm{eV}$ reported by Ulanski et al. [34] from the time of flight measurements for PVK films. It should be noted that the comparison of TL and transport data assumes that the energetic disorder, which governs the temperature dependence of charge mobility, does not change notably at low temperatures. Therefore TL method can give practically the same distribution of localized states and its analysis can yield an effective density of states width in quite good agreement with the values estimated from the charge carrier transport investigations [29].

Addition of 40 wt \% PBD to PVK results in a considerable change in the TL spectrum. The TL map, the extracted monochromatic and isothermal spectra for the PVK-PBD blend are presented in figure 5. The monochromatic TL curve for $\lambda=550 \mathrm{~nm}$ (figure $5 \mathrm{~b}$ ) has main maximum at $105 \mathrm{~K}$ and a shoulder in the low temperature range, which corresponds to the low-temperature maximum observed for neat PVK (figure 4b). A shift of the position of the main TL peak from $130 \mathrm{~K}$ for neat PVK to $105 \mathrm{~K}$ for PVK-PBD blend indicates that in the blend shallower traps are dominating than
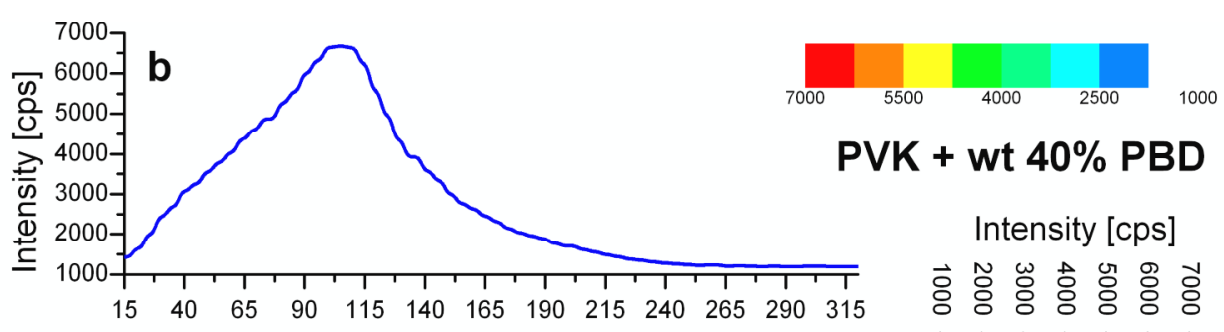

PVK + wt $40 \%$ PBD

Intensity [cps]

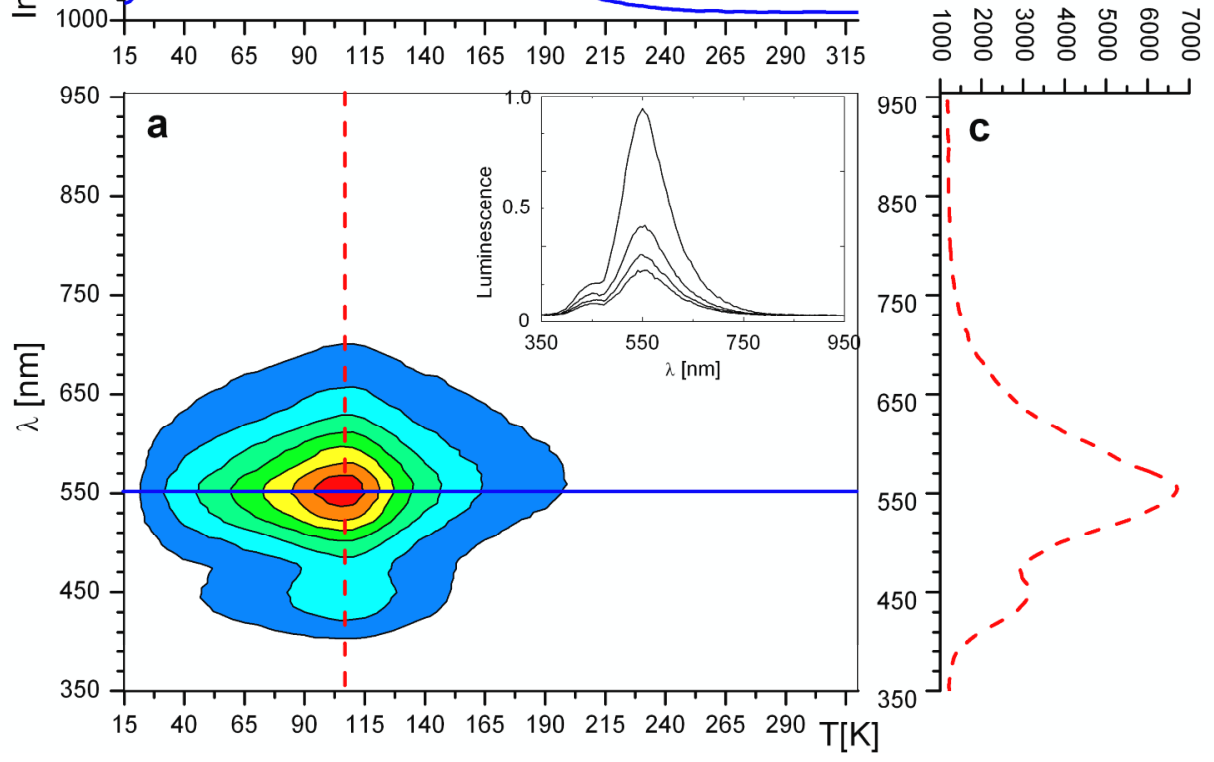

Figure 5. TL spectrum map for PVK-PBD (40 wt \%) blend film (a) with separated monochromatic TL curve (b) and spectral resolved curve (c). Marked lines on TL map indicate: chosen wavelength (horizontal line) and chosen temperature (vertical line). The inset shows spectra of isothermal luminescence decay at $15 \mathrm{~K}$ before TL run. 
those found in PVK. This is confirmed by the partial heating experiment for the blend which provides activation energy of about $150 \mathrm{meV}$ for charge carriers detrapping process close to the TL maximum at $105 \mathrm{~K}$. Similar value of trap depth was reported for PVK with $30 \mathrm{wt} \%$ PBD blend [17].

Figure 5c presents isothermal SRL spectra recorded at temperature close to the TL maximum (105 K). One can see two separated emission bands: a dominant one with a maximum at $550 \mathrm{~nm}$ and the second one with a maximum at $430-440 \mathrm{~nm}$. Very similar isothermal spectra were recorded at $15 \mathrm{~K}$ before starting the TL heating run (see an inset in figure $5 \mathrm{a}$ ).

The main maximum is shifted by $c a 20 \mathrm{~nm}$ towards longer wavelengths in comparison to that for PVK. Both emission bands in PVK-PBD blend can be attributed to the exciplexes formed between the carbazole group and PBD molecule. The dominant band with a maximum at $550 \mathrm{~nm}$ originates from the triplet exciplexes. The second one with maximum at $430-440 \mathrm{~nm}$ comes from the singlet exciplexes and/or monomeric triplet states of carbazole groups as discussed before.

The TL results evidentially indicate that introduction of PBD to PVK results in a reduction of deep trap contribution due to creation of shallow traps in a form of exciplexes on an expense of the excimers. This improves transport properties in comparison with neat PVK matrix.

\subsection{TL versus $E L$}

The comparison of the EL and SRTL spectra for the PVK and PVK-PBD systems (see figures 2, 4c and 5c) shows a variety of recombination states, which take part in radiative recombination processes in these phenomena. Therefore for next analysis and discussion of the results the spectra are presented together in figure 6.

In the EL spectrum for PVK the band connected with the singlet excimer states is dominating while the long-wavelength emission in $470-600 \mathrm{~nm}$ range is mainly attributed to the triplet excimer states and has lower intensity. Whereas the interrelationship between these bands in the SRTL spectrum is reversed; the long-wavelength band predominates definitely. For PVK-PBD system, a similar relation of the EL and the SRTL spectra is observed as described above but in this case both main bands are related to the exciplex states. Generally one can state that in PVK-PBD system the singlet exciplexes dominate in the EL emission (band at $420-430 \mathrm{~nm}$ ), while the radiative deactivation of triplet 


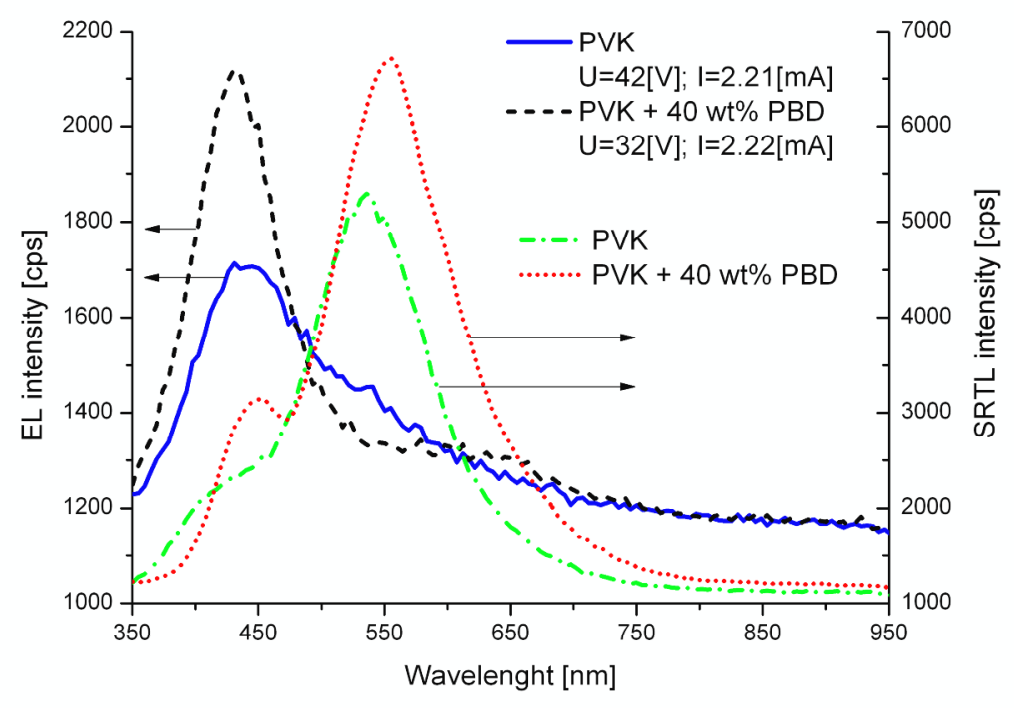

Figure 6. The comparison of electroluminescence spectra of single layer PLEDs (extracted from figure 2) and SRTL spectra registered at the TL maximum temperature for PVK and PVK-PBD (40 wt \%) films (extracted from figure $4 \mathrm{c}$ and from figure $5 \mathrm{c}$ ).

exciplexes (band at $550 \mathrm{~nm}$ ) results in TL spectra. Moreover the addition of PBD to PVK causes the reduction of EL emission in the range $470-600 \mathrm{~nm}$, what is related to the monomer and the excimer triplet states of carbazole groups, as discussed earlier. It results from two reasons: lower concentration of the carbazole groups in the blend and fact that the triplet exciplexes formation competes with a creation of the triplet excimers. The PBD molecules can cause capturing of carbazole group triplet excitons and simultaneously, the formed triplet exciplex can improve the transport process of charge carriers in the PVK-PBD blend as stated above.

For the mechanisms of radiative recombination observed in two phenomena (EL and SRTL) the temperature is determining factor. At low temperature (TL experiment conditions) the charge carriers, which are localized mainly on the exciplex site, have a long enough time to recombine radiatively by intersystem crossing. In the EL phenomenon carried out at room temperature the exciplex triplet sites do not act as radiative recombination centres any more but rather work as transport and/or the trapping sites with short residence time for charge carriers.

As it was mentioned in the introduction, the long-wavelength (above $c a 600 \mathrm{~nm}$ ) EL emission in PVK was related to different extended staking of carbazole groups [9] or to other species of recombination states present in PVK film [12]. However the triplet eximer of carbazole groups 
producing the broad emission band with maximum at $530 \mathrm{~nm}$ is known $[13,14]$ and has been demonstrated above. It is worth to underline that some authors undertake the trials to clear the origin of the emission at $600-750 \mathrm{~nm}$ range [9]. In majority cases the long-wavelength emission with small band about $600 \mathrm{~nm}$ has been observed in EL [12] and also in low temperature PL [9] for PVK film without previous, additional UV irradiation. It seems to be probable that the emission in range 600$750 \mathrm{~nm}$ can be related to a photochemical species. The photochemical species can be formed in small amount already during synthesis of PVK and/or preparation of the PVK films and they can influence on the observed photophysical processes. This hypothesis can be justified by the appearance of increased emission in range 600-750 $\mathrm{nm}$ in both EL and SRTL spectra (see figure 6) after introduction of PBD to PVK. This increase related to the new species can also be caused by improvement of balance between holes and electrons but with much lower contribution in comparison with the enhancement of the dominant band with maximum at $430 \mathrm{~nm}$. The contribution of this emission increases with increasing of driving voltage $[9,35]$ and it can be related to enhancement of oxidation processes by high electric field. Such phenomenon is observed especially for PLEDs prepared under ambient conditions.

\section{Conclusion}

In conclusion, we demonstrated that application of SRTL technique provides valuable information on the release and recombination of trapped charge carriers. The origin of the radiative recombination process producing the TL spectra can be identified by spectral resolved monitoring of the emitted light in 15-325 K temperature range. By detailed analysis of SRTL, PL and EL results it was possible to distinguish the excited states, trapping/transport sites and recombination centres existing in PVK and PVK-PBD electroluminescent matrices.

In neat PVK films, deep traps with depth about $200 \mathrm{meV}$ related to a triplet excimer dominate, while in PVK-PBD (40 wt \%) blend films the traps are attributed to a triplet exciplexes formed by the carbazole groups and PBD molecules constituting the main trap population. Depth of the traps in PVK-PBD blend is somewhat smaller than that in PVK. The analysis of the EL spectra shows that in PVK and PVK-PBD blend the dominating radiative centres are singlet excimer and singlet exciplex, 
respectively. However in neat PVK, some contributions of the triplet monomer and at least two kinds of triplet excimer states were also detected in electroluminescence. The triplet exciplexes which are formed at the cost of triplet excimers of the carbazole groups do not take part in the EL emission of PVK-PBD blend. The triplet exciplexes take part in radiative recombination in TL experiment and can take part in the transport of charge carriers in the PVK-PBD blend.

In spite of the fact that new conjugated polymers with large HOMO-LUMO separation are available, the PVK-PBD blend is still a good host matrix for low molecular weight emitters for single layer PLEDs with blue and white emission.

The SRTL is very useful technique for an identification of localized sites and recombination centres in materials used as active layers in organic LEDs.

\section{Acknowledgements}

The authors are grateful to Prof. J. Ulanski and Prof. J. K. Jeszka for helpful discussion.

\section{References}

[1] 2007 Organic light-emitting materials and devices ed Z Li and H Meng (Boca Raton: Taylor and Francis)

[2] Kalinowski J 2005 Organic Light-Emitting Diodes; Principles, Characteristics and Processes (New York: Marcel Dekker)

[3] Perepichka D F, Meng N and Ling M-M 2007 Phosphorescent polymer light-emitting diodes Organic light-emitting materials and devices, ed Z Li and H. Meng (Boca Raton: Taylor and Francis) pp 413-449

[4] Yang X-H, Jaiser F and Neher D 2008 Physical processes in polymer-based electrophosphorescent devices Highly efficient OLEDs with phosphorescent materials, ed H Yerisin (Weinhein: WILEYVCH Verlag GmbH \& Co. KgaA) pp 221-258

[5] Luszczynska B, Dobruchowska E, Glowacki I, Ulanski J, Jaiser F, Yang X, Neher D, Danel A 2006 Poly(N-vinylcarbazole) doped with a pyrazoloquinoline dye: a deep blue light-emitting composite for light-emitting diode applications J. Appl. Phys. 99 024505(1)-(4) 
[6] Kido J, Hongawa K, Okuyama K and Nagai K 1993 Bright blue electroluminescence from poly(Nvinylcarbazole) Appl. Phys. Lett. 63 2627-2629

[7] Hu B, Yang Z and Karasz F E 1994 Electroluminescence of pure poly(N-vinylcarbazole) and its blends with a multiblock copolymer J. Appl. Phys. 76 2419-2422

[8] D’Angelo P, Bara M, Cassinese A, Maglione M G, Vacca P, Minarini C, Rubino A 2007 Electrical transport properties characterization of PVK (poly N-vinylcarbazole) for electroluminescent devices applications Solid-State Electronics 51 123-129

[9] Qian L, Bera D and Holloway P H 2007 Electrophosphorescence from triplet excimers in poly(Nvinylcarbazole) Appl. Phys. Lett. 90 103511(1)-(3)

[10] Jiang X, Register R A, Killeen K A, Thompson M E, Pschenitzka F, Hebner T R and Sturm J C 2002 Effect of carbazole-oxadiazole excited-state complexes on the efficiency of dye-doped light-emitting diodes J. Appl. Phys. 91 6717-6724

[11] Beljonne D, Ye A, Shuai Z and Bredas J-L 2004 Chain-length dependence of singlet and triplet exciton formation rate in organic light-emitting diodes Adv. Func. Mater. 14 684-692

[12] Qian L, Bera D and Holloway P H 2007 White light emission from single layer poly-(Nvinylcarbazole) polymeric light-emitting devices by mixing singlet and triplet excimer emissions J. Chem. Phys. 127 244707(1)-(6)

[13] Burkhart R D and Chakraborty D K 1990 Binding energies of triplet excimers in poly(Nvinylcarbazole) solid films from laser-based kinetic spectroscopy between 15 and $55 \mathrm{~K}$ J. Phys.

Chem. 94 4143-4147

[14] Glowacki I, Ulanski J and Kozankiewicz B 1998 Charge carrier detrapping in PVK films as seen by thermoluminescence after excitation at $15 \mathrm{~K}$ Space charge in solid dielectrics ed J C Fothergill and L A Dissado (Leicester, UK: The Dielectrics Society) pp 251-258

[15] Sainova D, Neher D, Dobruchowska E, Luszczynska B, Glowacki I, Ulanski J, Nothofer H-G, Scherf U 2003 Thermoluminescence and electroluminescence of annealed polyfluorene layers Chem. Phys. Lett. 371 15-22

[16] Schmechel R and von Seggern H 2004 Electronic traps in organic transport layers phys. stat. sol.

(a) 201 1215-1235 
[17] Luszczynska B, Dobruchowska E, Glowacki I, Danel A, Ulanski J 2009 Thermoluminescence of the blue light-emitting system based on poly(9-vinylcarbazole) doped with a pyrazoloquinoline dye J. Lumin. 129 1215-1218

[18] Klöpffer W 1969 Transfer of Electronic Excitation Energy in Polyvinyl Carbazole J. Chem. Phys. $502337-2343$

Klöpffer W, Fischer D 1973 Triplet Energy Transfer in Solid Solutions and Films of Polyvinyl Carbazole J. Pol. Sci. Part C 40 43-56

[19] Bässler H 1984 Charge transport in molecularly doped polymers Philos. Mag. B 50 347-362

[20] Johnson G E and Good T A 1982 Molecular weight dependent emission properties of poly(Nvinycarbazole) in polymer blends Macromolecules 15 409-417

[21] Itaya A, Okamoto K, Kusabayashi S 1976 Emission spectra of the vinyl polymers with pedant carbazolyl groups Bull. Chem. Soc. Jpn. 49 2082-2088

[22] Gong X, Lim S-H, Ostrowski J C, Moses D, Bardeen Ch J, Bazan G C 2004 Phosphorescence from iridium complexes doped into polymer blends J. Appl. Phys. 95 948-953

[23] Negres R A, Gong X, Ostrowski J C, Bazan G C, Moses D and Heeger A J 2003 Origin of efficient light emission from a phosphorescence polymer/organometallic guest-host system Phys. Rev. B 68 115209(1)-(8)

[24] Glowacki I and Ulanski J 1995 Simultaneous thermoluminescence and thermally stimulated currents measurements in poly(N-vinylcarbazole)/polycarbonate blends J. Appl. Phys. 78 10191025

[25] Dobruchowska E, Okrasa L, Glowacki I, Ulanski J, Boiteux G 2004 The 'wet dog' effect in polymers as seen by thermoluminescence Polymer $\mathbf{4 5}$ 6027-6035

[26] Akuetey G and Hirsh J 1989 Thermal depolarization measurements on PVK films, J. Phys. D Appl. Phys. 22 174-181

[27] Pochan J M, Hilman D F and Nash R 1975 Dielectric relaxation studies of poly(Nvinylcarbazole) and poly(3-chloro-N-vinylcarbazole) including the effects of sorbed oxygen $J$. Appl. Phys. 46 4115-4119 
[28] Arkhipov V I, Emalianova E V, Kadashchuk A, Blonsky I, Nespurek S, Weiss D S, Bässler H 2002 Polaron effects on thermally stimulated photoluminescence in disordered organic systems Phys. Rev. B 65 165218(1)-(8)

[29] Fishchuk I I, Kadashchuk A K, Vakhin A, Korosko Y, Bässler H, Souharce B and Scherf U 2006 Transition from trap-controlled to trap-to- trap hoping transport in disordered organic semiconductors Phys. Rev. B 73 115210(1)-(11)

[30] Movaghar B, Ries B and Grunewald M 1986 Diffusion and relaxation of energy in disordered systems: Departure from mean-field theories Phys. Rev. B 34 5574-5582

[31] Arkhipov V I, Emalianova E V, Kadashchuk A and Bässler H 2001 Hopping model of thermally stimulated photoluminescence in disordered organic materials Chem. Phys. 266 97-108

[32] Bässler H 1994 Exciton and charge carrier transport in random organic solids Disordered effect on relaxation processes ed R Richert and A Blumen (Berlin: Springer-Verlag) pp 485-507

[33] Bässler H 1981 Lokalized states and electronic transport in single component organic solids with diagonal disorder phys. stat sol. (b) 107 9-54

[34] Ulanski J, Sielski J, Glowacki I, M. Kryszewski M 1992 Transition from Dispersive to Nondispersive Hole Transport in Poly-N-vinylocarbazole/ Polycarbonate Mixtures, IEEE Transactions on Electrical Insulation 27 714-718

[35] Yap C C, Yahaya M, Salleh M M 2008 The effect of driving voltage on the electroluminescence property of a blend of poly(9-vinylcarbazole) and 2-(4-biphenylyl)-5-phenyl-1,3,4-oxadiazole Curr. Appl. Phys. 9 1038-1041 


\section{Figure captions}

Figure 1. Chemical structures of investigated materials and diagram of energetic levels. The values of energetic levels according to ref. 4.

Figure 2. Normalized photoluminescence spectra of PVK and PVK-PBD (40 wt \%) thin films under excitation at $337 \mathrm{~nm}$ and electroluminescence spectra of single layer PLEDs prepared based on PVK and PVK-PBD (40 wt \%) blend, measured for the same current density, $22 \mathrm{~mA} / \mathrm{cm}^{2}$. The inset shows the corresponding absorption spectra.

Figure 3. Three-dimensional thermoluminescence spectra for neat PVK film registered under the heating rate $7 \mathrm{~K} / \mathrm{min}$ after excitation by $\mathrm{HBO}-200$ lamp with band filter at $15 \mathrm{~K}$.

Figure 4. TL spectrum map for neat PVK film (a) with separated monochromatic TL curves (b) and spectral resolved curves (c). Marked lines on TL map indicate: chosen wavelengths (horizontal lines) and chosen temperatures (vertical lines). The inset presents spectra of isothermal luminescence decay at $15 \mathrm{~K}$ before TL run.

Figure 5. TL spectrum map for PVK-PBD (40 wt \%) blend film (a) with separated monochromatic TL curve (b) and spectral resolved curve (c). Marked lines on TL map indicate: chosen wavelength (horizontal line) and chosen temperature (vertical line). The inset shows spectra of isothermal luminescence decay at $15 \mathrm{~K}$ before TL run.

Figure 6. The comparison of electroluminescence spectra of single layer PLEDs (extracted from figure 2) and SRTL spectra registered at the TL maximum temperature for PVK and PVK-PBD (40 wt $\%)$ films (extracted from figure $4 \mathrm{c}$ and from figure $5 \mathrm{c}$ ). 


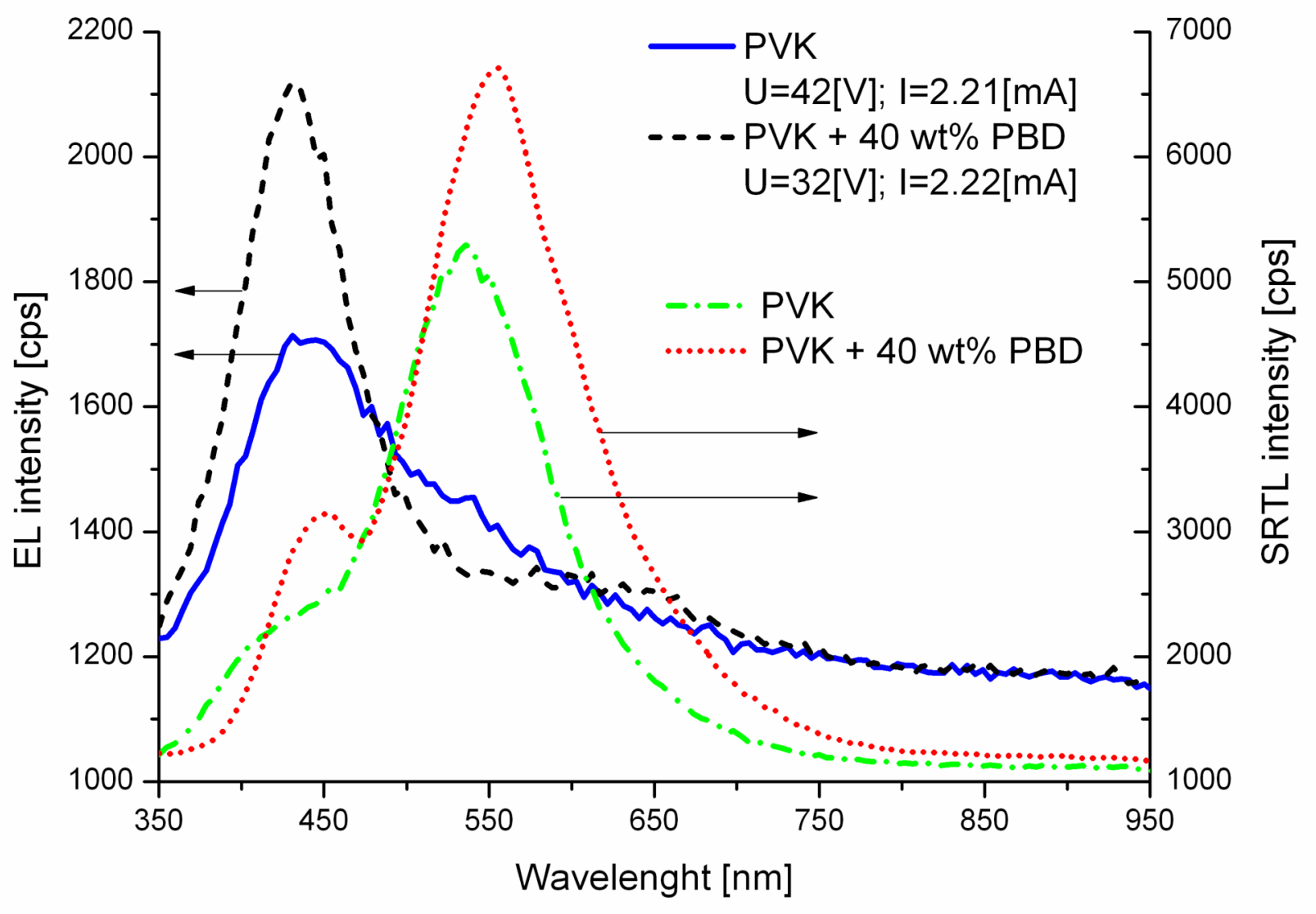

Figure 1 (Figure6_Glowacki_2010.TIF) 